\title{
Exogenous and endogenous response priming with auditory stimuli
}

\author{
Peter E. Keller ${ }^{1}$ and Iring Koch ${ }^{2}$ \\ ${ }^{1}$ Max Planck Institute for Human Cognitive and Brain Sciences, Leipzig, Germany \\ 2 RWTH Aachen University, Institute of Psychology, Aachen, Germany
}

Received 16.07.2006

Accepted 28.11.2006

\section{Keywords}

priming, stimulus-response compatibility, response-effect compatibility, auditory perception, auditory imagery

\begin{tabular}{|c|c|}
\hline ABSTRACT & $\begin{array}{l}\text { uli, and hence effects of REC on response times } \\
\text { reflect the anticipation of forthcoming tones. Re- }\end{array}$ \\
\hline $\begin{array}{l}\text { Exogenous and endogenous response priming } \\
\text { were investigated by comparing performance on } \\
\text { stimulus-response compatibility (SRC) and re- } \\
\text { sponse-effect compatibility (REC) tasks using a } \\
\text { repeated measures design. In the SRC task, par- } \\
\text { ticipants made finger taps at high or low locations }\end{array}$ & $\begin{array}{l}\text { sults indicated that response times were shorter } \\
\text { with compatible mappings between tones and } \\
\text { responses than with incompatible mappings in } \\
\text { both tasks. Although these SRC and REC effects } \\
\text { did not differ reliably in magnitude, they were } \\
\text { uncorrelated across participants. Thus, although }\end{array}$ \\
\hline $\begin{array}{l}\text { in response to centrally presented visual stimuli } \\
\text { paired with high- or low-pitched tones. In the } \\
\text { REC task, the tones were triggered by responses } \\
\text { instead of being presented with the visual stim- }\end{array}$ & $\begin{array}{l}\text { exogenous and endogenous response priming } \\
\text { may be functionally equivalent at the level of the } \\
\text { group, it is unclear whether this is the case at } \\
\text { the level of the individual. }\end{array}$ \\
\hline
\end{tabular}

\section{INTRODUCTION}

Voluntary actions are usually preceded by thoughts prompted by events in the environment. However, the link between environmental events and the thoughts that they apparently inspire can vary in terms of directness. Sometimes thoughts match events directly, as is the case, say, when a mental representation of the first tones of the Pink Panther theme is activated by hearing a busker play the music. At other times, thoughts are evoked less directly through learnt associations between environmental events, as is the case when a visual image of the pink feline himself activates a representation of the theme music. Regardless of whether thoughts are evoked via direct or indirect routes, they can lead to action, e.g., humming in the above scenarios.

The notion of direct and indirect routes to action is to some extent reflected in the distinction between experimental paradigms that manipulate stimulusresponse compatibility (SRC) and response-effect

compatibility (REC). SRC refers to the degree of correspondence between features of a stimulus and features of its target response on concrete physical and/or abstract conceptual dimensions (e.g., Fitts \& Deininger, 1954; Kornblum, Hasbroucq, \& Osman, 1990). REC refers to the degree of correspondence between features of a response and the effects that it produces in the environment (Hoffmann, Stoecker, \& Kunde, 2004; Koch \& Kunde, 2002; Kunde, 2001).

It is typically found in SRC tasks that choice reaction times are faster when stimuli and responses are compatible than when they are incompatible (for reviews see Proctor \& Reeve, 1990, and Hommel \& Prinz, 1997). For example, left key presses are initiated more quickly than right key presses in response to a visual stimulus that appears on a person's left hand side, and vice versa

Correspondence concerning this article should be addressed to Peter Keller, Max Planck Institute for Human Cognitive and Brain Sciences, Department of Psychology, Stephanstr. 1a, D-04103 Leipzig, Germany, tel.: +49 341 9940-234, fax: +49 341 9940-204, e-mail: keller@cbs.mpg.de 
(Fitts \& Deininger, 1954). Such SRC effects occur even when the location of the stimulus is ostensibly irrelevant to the task, which may involve responding to another stimulus dimension such as color (Simon, 1969). REC effects are superficially similar to SRC effects, but they are elicited under conditions where stimuli are totally arbitrary (both on relevant and irrelevant dimensions), and only the correspondence between responses and their forthcoming effects is manipulated. For example, when responding to centrally presented colored stimuli, left key presses are initiated more quickly if they reliably trigger the appearance of objects located on the left than if they produce effects on the right, and vice versa (Kunde, 2001). Such REC results suggest that the distal effects of a response are brought to mind, presumably through a process of imagery, before movement is initiated.

The findings of studies employing SRC and REC paradigms can be interpreted parsimoniously in light of the ideo-motor approach to action, which postulates that movements arise through the anticipation of associated sensory effects (e.g., Greenwald, 1970; Harleß, 1861; James, 1890; Lotze, 1852; Prinz, 1987; see Hoffmann et al., 2004, and Koch, Keller, \& Prinz, 2004, for reviews). Thus, SRC effects occur because responses are primed exogenously by the perception of external stimulus events (e.g., Hommel, Müsseler, Aschersleben, \& Prinz, 2001; Hommel \& Prinz, 1997; Kunde, 2004), and REC effects derive from the endogenous priming of responses by imagined events (Kunde, 2003; Kunde, Koch, \& Hoffmann, 2004). In both cases it is typically assumed that while response selection and initiation benefit from such priming in the case of compatible mappings, costs are incurred when the need arises to inhibit inappropriate responses primed by incompatible (perceived or imagined) events (Kornblum et al., 1990; Kunde et al., 2004).

The fact that SRC and REC effects can be explained in terms of the same general mechanism (response priming) and have been invoked in support of the same theoretical (ideo-motor) principle suggests that these two compatibility phenomena may be intimately related. This would be consistent with the view that perception and imagery are functionally equivalent in terms of their influences upon action (see Finke, 1980, 1985; Hubbard \& Stoeckig, 1992; Intons-Peterson, 1992; Kosslyn, Ganis, \& Thompson, 2001; Tlauka \& McKenna, 1998). If so, then compatibility effect sizes should be similar across wellmatched SRC and REC tasks. Furthermore, the temporal dynamics of exogenous and endogenous response priming should be similar. The analysis of reaction time distributions (see Ratcliff, 1979) in SRC and REC studies has suggested that this is the case under certain circumstances. For example, Adam, Boon, Paas, \& Umiltà (1998) observed an SRC effect - the up-right/down-left advantage for vertically oriented stimuli and horizontally oriented responses - that grew with increasing reaction time. Adam et al. attributed this growth over time to the slow formation of verbal stimulus codes underlying the effect. The REC effects observed by Kunde (2001) - based on the correspondence between responses and their effects in terms of either spatial location or intensity - also grew with increasing reaction time. Kunde suggested that this pattern arose because the endogenous activation of anticipated effect codes (which are not necessarily verbal) is time consuming. Thus, SRC and REC effects may display similar time courses for different reasons.

Here it should be noted that the temporal dynamics of SRC effects appear to be context dependent. In the Adam et al. (1998) study cited above, SRC was manipulated on a response-relevant stimulus dimension (spatial orientation), and compatibility conditions were blocked. SRC effects based on information from irrelevant stimulus dimensions in mixed designs - as in the Simon (1969) task - typically show the opposite pattern to that observed by Adam et al. (1998), and decrease in magnitude with increasing reaction time (e.g., De Jong, Liang, \& Lauber, 1994; but see Wiegand $\&$ Wascher, 2005). This decrease can be taken to suggest that exogenous priming by perceived stimuli weakens over time as codes related to the irrelevant stimulus dimension decay. As far as we are aware, it is presently unknown whether the different temporal dynamics observed with SRC manipulations targeting relevant versus irrelevant stimulus dimensions is due to relevance per se or to differences in the trial-to-trial predictability of stimulus-response mappings in mixed versus blocked designs. The current study was not designed to address this question.

The current study investigates the degree to which exogenous and endogenous response priming are functionally equivalent - i.e., produce similar externally observable effects on behaviour - by examining the relationship between performance on closely matched auditory SRC and REC tasks in a repeated measures experimental design (see Figure 1). Our SRC task involved making single finger taps on keys at high or low spatial locations in response to two colored stimuli, each of which was paired with a high- or a low-pitched tone. In a compatible condition, high re- 
Compatible

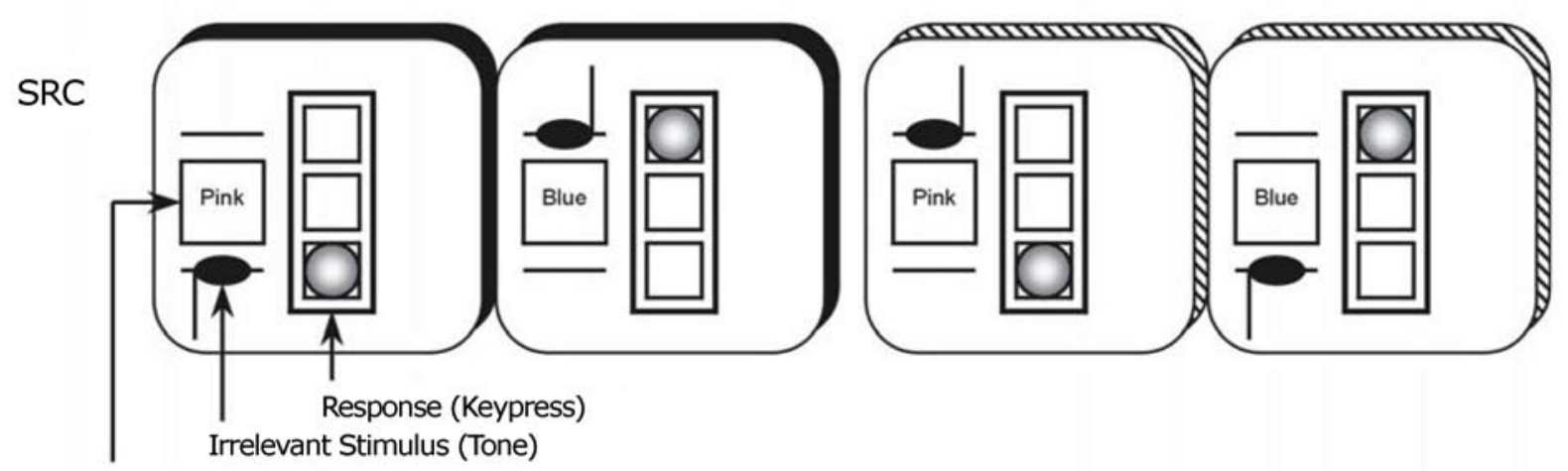

Imperative Stimulus (Color Patch)

REC

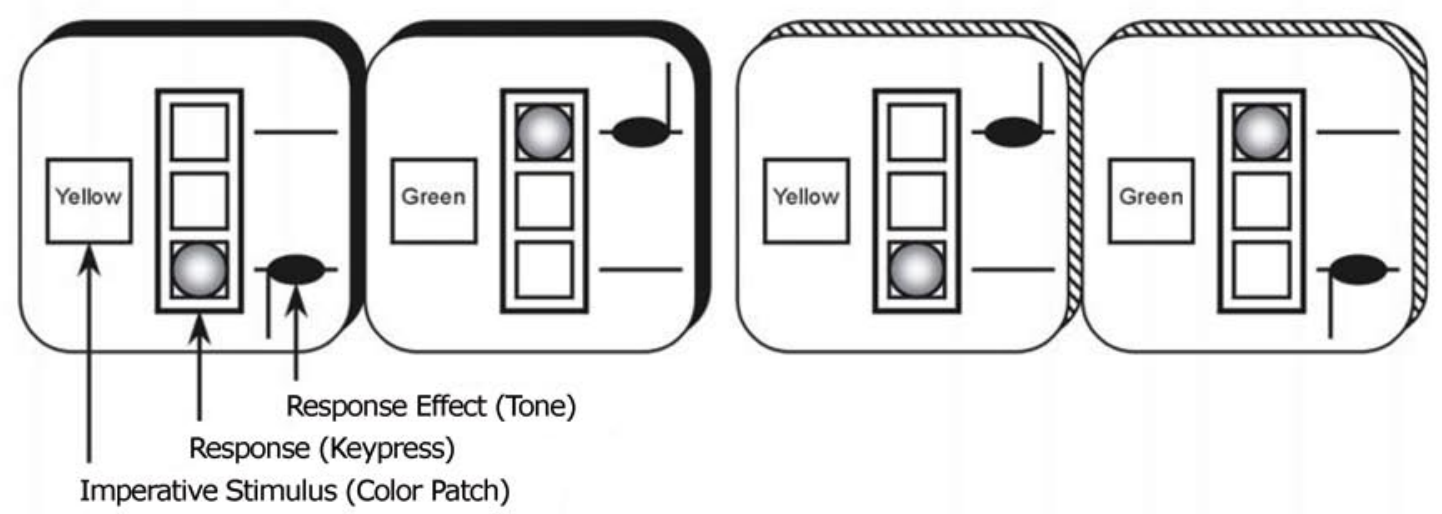

\section{Figure 1.}

Schematic diagram illustrating the combination of tasks (SRC vs. REC) and compatibility conditions (compatible vs. incompatible) in the experimental design. Each compartment in the diagram shows events from a single experimental trial. The SRC task and the REC task are similar insofar as they involve responding to an imperative visual stimulus by making a key press at a high or a low spatial location (different stimulus colors are used in the two tasks). The main difference between the SRC task and the REC task lies in the role of the high-and low-pitched tones (depicted at high vs. low spatial locations in the Figure, respectively), which serve as irrelevant stimuli in the SRC task and as irrelevant response effects triggered by the key presses in the REC task.

sponses were required to a color that was paired with the high tone and low responses were required to a color paired with the low tone. These contingencies were reversed in an incompatible condition. Research using similar SRC manipulations has supported the assumption that there is a conceptual correspondence between the dimensions of pitch height and spatial height (Lidji, Kolinsky, Lochy, Karnas, \& Morais, 2005; Rusconi, Kwan, Giordano, Umiltá, \& Butterworth, 2006; Simon, Mewaldt, Acosta, \& Hu, 1976). Our REC task was identical to our SRC task with the exception that the high and low tones were triggered by the finger taps instead of being presented simultaneously with the colored stimuli.

It was assumed that, under the above conditions, any observed compatibility effects would be attributable to the activation of mental representations of the tones via perception (SRC) or imagery (REC). These tone representations may prime high and low responses due to semantic overlap ('high/low' verbal labels; see Melara \& Marks, 1990) or pure spatial associations (pitches arranged vertically in mental representational space; see Rusconi et al., 2006). Our study was not designed to distinguish between these linguistic and non-linguistic types of priming, but rather just to distinguish between priming by perceived versus imagined auditory stimuli.

We assume that if exogenous and endogenous priming are functionally equivalent, then SRC and REC effects should be similar in magnitude and time course, and correlated with one another across individuals. Although we expected this to be the case, we also considered that it was possible that SRC effects may be larger than REC effects because exogenous priming is based on salient and unambiguous external stimuli while endogenous priming relies on the generation of mental images. Thus, REC effects might be relatively variable because individuals differ considerably in imagery abilities (Kosslyn, Brunn, Cave, \& Wallach, 1984; Richardson, 1999). 


\section{METHOD}

\section{Participants}

Forty healthy young adults ( 32 female and 8 male; mean age $=23.6$ years, $S D=3.7$ years) participated in the experiment. All but two participants preferred to respond with the right hand. Although half of the participants had musical experience (5-24 years of regular instrumental playing; mean $=12.0, \mathrm{SD}=6.2$ ), this experience did not influence the magnitude of the SRC and REC effects reliably. Therefore, we report combined results for musicians and nonmusicians in this article.

\section{Materials}

Visual stimuli consisted of four $16 \times 16 \mathrm{~mm}$ patches of color presented in the centre of a computer screen: pink, blue, yellow, or green. Auditory stimuli and response effects consisted of tones presented in a marimba timbre at a comfortable loudness level over PRO-10 earphones. Two different tone pitches were used: low $(392 \mathrm{~Hz})$ and high $(784 \mathrm{~Hz})$. All tones had a sharp onset followed by natural decay lasting approximately $500 \mathrm{~ms}$. The response box was $250 \mathrm{~mm}$ high and $150 \mathrm{~mm}$ wide, and had three metal plates embedded along the centre of the vertical plane of its surface. The plates, which served as response keys, measured $30 \times 30 \mathrm{~mm}$ and were separated from one another by $15 \mathrm{~mm}$. Stimulus presentation and response registration were controlled by ERTS software (BeriSoft Cooperation) running on a PC.

\section{Design and procedure}

A $2 \times 2$ repeated measures design was employed with the variables task (SRC, REC) and compatibility condition (compatible, incompatible). Task and compatibility condition order were counterbalanced, as described below.

Participants sat at a viewing distance of approximately $750 \mathrm{~mm}$ from the computer monitor, which rested on a table. The response box was positioned on a stand between the participant and the table at a comfortable height for tapping. The box was angled so that its surface was off-vertical by approximately 30 degrees. Half of the participants encountered the SRC task before the REC task, and the other half did the tasks in the reverse order. Participants received both written and oral instructions immediately before commencing each task.

The SRC task involved responding as quickly and accurately as possible to the pink and blue visual stim- uli by making single finger taps on the top and bottom response keys, respectively. Each participant was allocated randomly to one of two possible stimulusresponse assignments: pink-top/blue-bottom or pinkbottom/blue-top. At the start of each trial, a fixation cross appeared at the centre of the computer screen. The participant placed the index finger of his or her preferred hand on the middle 'home' key when ready. After a $1 \mathrm{~s}$ interval, the pink or blue stimulus appeared simultaneously with a high or low tone, depending on compatibility condition. The visual stimulus remained on screen until a response was registered. An error message appeared for $500 \mathrm{~ms}$ at the centre of the screen following incorrect responses. A blank screen intervened for $1500 \mathrm{~ms}$ between trials.

In the compatible condition, participants were required to respond to the color that was paired with the high tone by tapping on the top key, and to respond to the color paired with the low tone by tapping the bottom key. This mapping between tones and responses was reversed in the incompatible condition. There were two consecutive blocks of 60 test trials per compatibility condition. The first block of each compatibility condition was preceded by a practice block comprising 12 trials. The order in which compatibility conditions were run was counterbalanced across participants.

The procedure in the REC task was identical to the SRC procedure in all but two respects. First, the color patch stimuli were yellow and green rather than pink and blue. This change was implemented to prevent associations between colors and tones from being carried over from the SRC task to the REC task when tasks were encountered in this order. Stimulus color sets were not counterbalanced across tasks because we had no reason to expect that compatibility effects based on pitch and spatial height would be affected by the color of the imperative stimuli (none of our participants reported synaesthesia). Second, tones were triggered by taps on the top and bottom keys instead of being presented simultaneously with the visual stimuli. Thus, top key responses were paired with high tones and bottom key responses were paired with low tones in the compatible condition, while the incompatible condition used the reverse mapping.

Individual participants encountered the compatibility conditions in the same order for the SRC and the REC task. The instructions for both tasks specified that the tones are irrelevant and should be ignored. Although participants may have benefited from defying these instructions in the compatible SRC condition, it was considered important that the SRC and REC tasks were matched in terms of instructions and the blocking of compatibility 
conditions. Thus, our SRC task differs from conventional SRC tasks involving combined imperative and irrelevant stimuli in that trials for each compatibility condition were blocked in our procedure, whereas compatibility conditions are typically mixed. Note that it was necessary to present compatible trials and incompatible trials in separate blocks in the REC task because otherwise it would have been impossible for participants to anticipate the response-effect tones reliably.

\section{RESULTS}

\section{Response times}

Reaction time was measured as the time taken to lift the tapping finger from the home key following stimulus onset, and movement time was defined as the time it took for the finger to travel to the response key. Trials with reaction times shorter than $100 \mathrm{~ms}$, and reaction times or movement times longer than $1500 \mathrm{~ms}$, were classified as outliers $(1.88 \%$ of trials in which a correct response was made) and excluded from analysis. Likewise, reaction times and movement times for trials in which an incorrect response was made ( $1.11 \%$ of trials) were not analyzed. Reaction times and movement times from test blocks were entered into separate $2 \times 2 \times 2 \times 2$ analyses of variance (ANOVAs) with the within-participants variables of task and compatibility condition and the between-participants variables of task order (SRC-REC vs. REC-SRC) and compatibility condition order (compatible-incompatible vs. incompatible-compatible). The criterion for statistical significance was set at $\alpha=.05$. Similar patterns of results were observed for reaction time and movement time, so for the analyses reported below we combined these measures by summing to produce a single response time measure. Our task order and compatibility condition order groups were fairly well matched in terms of overall performance: The main effects and interaction of these variables were not statistically significant, ps $>.25$.

Response times were on the whole similar for the SRC task and the REC task. Indeed, the main effect of task in the ANOVA on response times was not significant, $F(1,36)=1.89, p=.18, \eta_{p}^{2}=.05$. However, there was a reliable interaction between task and task order, $F(1,36)=8.84, p<.01, \eta_{p}^{2}=.20$. Participants who started with the SRC task experienced only a small (14 $\mathrm{ms}$ ) reduction in mean response time when moving from one task to the next (SRC $=561 \mathrm{ms,}$ REC $=547 \mathrm{~ms}$ ), while participants who started with the REC task experienced a larger $(38 \mathrm{~ms}$ ) reduction in mean response time $(\mathrm{REC}=535 \mathrm{~ms}, \mathrm{SRC}=497$

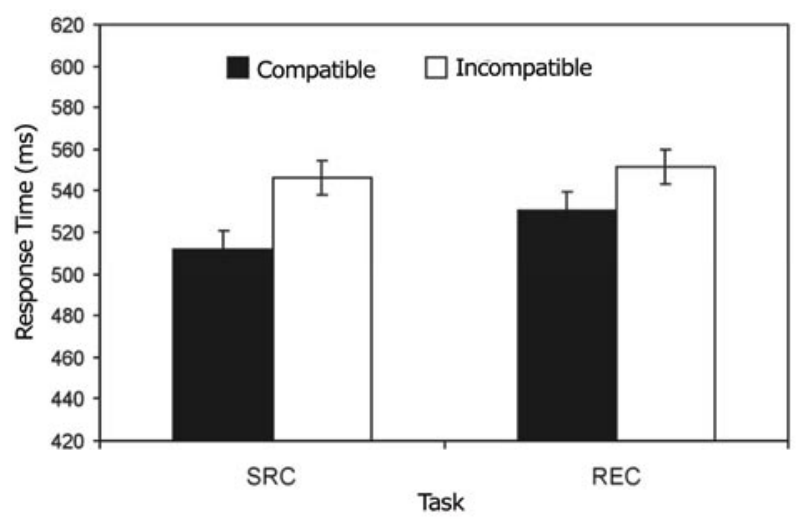

Figure 2.

Mean response times in compatible and incompatible conditions for the SRC task and the REC task. Error bars indicate $95 \%$ confidence intervals (computed according to the method described by Masson \& Loftus, 2003).

$\mathrm{ms})$. We are unsure why this was the case. Perhaps in the REC-SRC order group, associations formed between movements and effect tones during the REC task led to especially potent response priming by these tones when they were subsequently encountered in the SRC task (see Elsner \& Hommel, 2001, 2004).

Notwithstanding the above interaction, the notion that performance was on the whole commensurate for the two tasks was supported by a strong positive cor relation between participants' mean response times on the SRC task and their mean response times on the REC task, $r=.84, \mathrm{~N}=40, p<.001$. This relationship remained strong when correlations were computed separately for participants who started with the SRC task $(r=.83, \mathrm{~N}=20, p<.001)$ and those who started with the REC task $(r=.94, \mathrm{~N}=20, p<.001)$. The correlation coefficients for these two groups were not significantly different from one another, $p=.11$.

Figure 2 shows average response times in compatible and incompatible conditions for the SRC and REC tasks. Importantly, participants were on average slower in incompatible conditions than in compatible conditions. The reliability of this result was confirmed by a significant main effect of compatibility condition in the ANOVA, $F(1,36)=16.81, p<.001, \eta_{p}^{2}=.32$. Although the compatibility effect (incompatible minus compatible response times) is numerically larger for the SRC task (34 ms) than for the REC task ( $21 \mathrm{~ms}$ ), the absence of a significant interaction between compatibility condition and task in the ANOVA suggests that this difference is not reliable, $F(1,36)=1.69$, $p=.20, \mathrm{n}_{\mathrm{p}}^{2}=.05$. This was the case irrespective of the order in which tasks and compatibility conditions were presented, $p s>.29$.

Given the numerical difference in SRC and REC effects noted above, we ran separate ANOVAs on the 

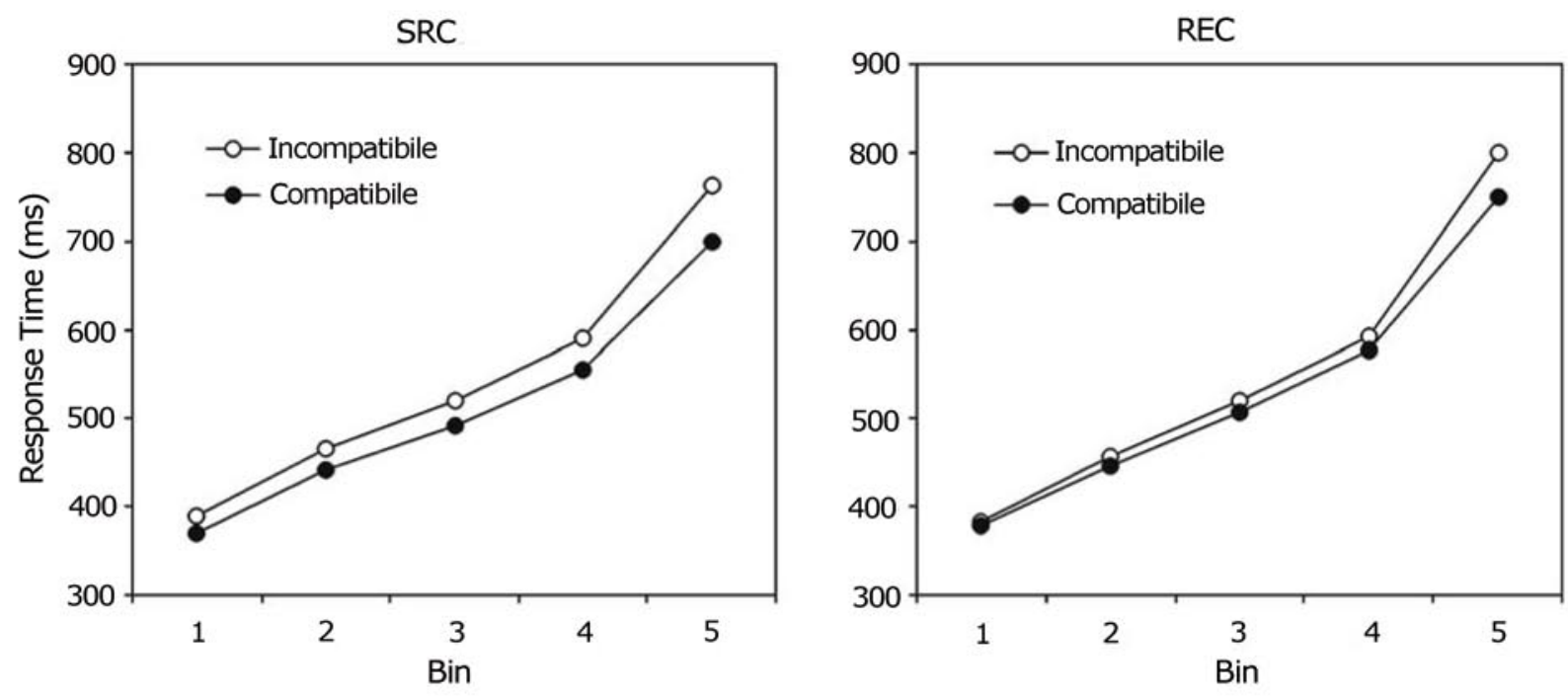

Figure 3.

Mean response times in each quintile bin for compatible and incompatible conditions from the SRC task and the REC task.

SRC and REC data to test whether each compatibility effect itself is reliable. Although the effect size is larger for SRC than for REC, both analyses yielded a statistically significant main effect of compatibility condition: $\operatorname{SRC} F(1,36)=20.84, p<.001, \eta_{p}^{2}=.37$; $\operatorname{REC} F(1,36)=4.97, p<.05, \eta_{p}^{2}=.12$. However, virtually zero correlation was observed between the sizes of the SRC and REC effects across participants ( $r=-.01, \mathrm{~N}=40)$, suggesting that these effects were independent of one another. This was also the case when correlations were computed separately for participants who started with the SRC task $(r=-.13$, $\mathrm{N}=20$ ) and those who started with the REC task ( $r=0.06, \mathrm{~N}=20$ ). The correlation coefficients for these two groups were not significantly different from one another, $p=.56$.

Finally, to compare the temporal dynamics of SRC and REC effects, we conducted a distribution analysis on response times. Each participant's SRC and REC response time distribution was computed separately for compatible and incompatible conditions. The average response time in each quintile 'bin' of these distributions was then calculated. Figure 3 shows the binned response time data in compatible and incompatible conditions for the SRC and REC tasks.

These data were entered into a $2 \times 2 \times 5$ repeated measures ANOVA with variables task, compatibility condition, and bin (with the Greenhouse-Geisser correction applied when the degrees of freedom numerator exceeded one). Apart from main effects of compatibility condition, $F(1,39)=15.88, p<.001$, $\eta_{p}^{2}=.29$, and bin, $F(4,156)=370.32, p<.001$, $\eta_{p}^{2}=.91$, this analysis revealed significant interactions between task and bin, $F(4,156)=6.38$, $p<.02, \eta_{p}^{2}=.14$, and compatibility condition and bin, $F(4,156)=6.06, p<.02, \eta_{p}^{2}=.14$. As can be seen in Figure 3, the interaction between task and bin indicates that the slowing of response times with bin was more pronounced in the REC task than in the SRC task. In other words, fast responses had similar absolute durations in both tasks, whereas slow responses were slower in the REC task than in the SRC task. More importantly, as can be seen in Figure 3, the compatibility effect grew with increasing response time in both tasks. Paired comparisons within each bin revealed that SRC effects were significant in all bins and REC effects were significant from the second bin on (one-tailed $p s<.05$ ). These findings suggest that the temporal dynamics of our SRC and REC effects were similar. Indeed, the three-way interaction between task, compatibility condition, and bin was not significant, $F(1,39)<1$.

\section{Error rates}

The pattern of results for errors rates matched the main response time results. Participants made similar amounts of errors on average for the SRC task (1.08\%) and the REC task (1.15\%), and slightly more errors were made for both tasks in incompatible conditions (1.22\%) than in compatible conditions (1.01\%). An ANOVA on these data revealed that the main effects of task and compatibility condition, and the interaction between these variables, were not statistically significant.

\section{DISCUSSION}

Exogenous and endogenous response priming were compared by selectively manipulating the compatibil- 
ity between stimuli and responses in an SRC task, and responses and their effects in an REC task, respectively. Both tasks required participants to make finger taps at high and low spatial locations in response to arbitrary visual stimuli. High- and low-pitched tones, which were ostensibly irrelevant to the tasks, were presented simultaneously with the visual stimuli in the SRC task and simultaneously with the responses in the REC task. Mean response times for the SRC and REC task were similar in magnitude, and were positively correlated with one another across participants. Thus, the two tasks were approximately matched in difficulty. Moreover, for both tasks it was found that response times were shorter in blocks of trials where the mapping between tones and responses was compatible (on the 'height' dimension) than in blocks where this mapping was incompatible. The magnitudes of the SRC and REC effects did not differ reliably, and therefore the hypothesis that exogenous response priming by perceived tones and endogenous response priming by imagined tones are similar in potency cannot be rejected based on our results. Furthermore, SRC and REC effects both grew with increasing response time, suggesting that the temporal dynamics of exogenous and endogenous priming were similar. Nevertheless, SRC and REC effects were uncorrelated across participants: People who displayed relatively large SRC effects did not necessarily have large REC effects.

The current findings provide evidence that movements to high spatial locations were primed both by the perception (SRC) and by the anticipation (REC) of high-pitched tones, and, conversely, movements to low spatial locations were primed both by perceived and by anticipated low-pitched tones. Note that the influence of REC on response times is assumed to indicate that participants anticipated upcoming tones because these tones were triggered only by the completion of the response in each trial (see Kunde, 2001). This REC effect is itself noteworthy as it provides evidence that pitch-height compatibility affects movement even when tones are imagined rather than perceived.

The above interpretation assumes that movements were primed by the activation of mental representations of tones in both tasks. These representations were activated directly by the 'irrelevant' tones presented simultaneously with the imperative visual stimuli in the SRC task, while they were activated indirectly via learnt associations between the visual stimuli and the response-effect tones in the REC task. Consistent with work on action-effect learning (Elsner \& Hommel, 2001, 2004; Elsner et al., 2002; Ziessler, Nattkemper, \& Frensch, 2004), it is assumed here that these as- sociations were acquired during the initial trials in each experimental condition.

The finding that SRC and REC effect magnitudes and temporal dynamics were not significantly different from one another suggests that exogenous and endogenous priming are functionally equivalent at the level of the group (i.e., when effects are averaged across participants), while the finding that SRC and REC effects were uncorrelated suggests that this equivalence may not extend down to the level of the individual. Thus, the present results imply that individuals may differ with regard to whether they are more susceptible to response priming based upon perceived events or imagined events. This suggests that interindividual variations in SRC and REC effect magnitude might have different sources.

Factors that may have contributed to our SRC effect include pitch perception abilities and the potency of the pitch-height stereotype in the individual. Indeed, the association of pitches with 'high' and 'low' verbal labels and/or spatial locations is not as uniform in the population as is often assumed (Neuhoff, Knight, \& Wayand, 2002; Walker, 1987). The magnitude of our REC effect, while also relying on the pitch-height stereotype, was presumably more intimately related to auditory imagery abilities than perceptual abilities. Therefore, it may be the case that the observed independence of SRC and REC effects arose because variability in the former was dominated by individual differences in the potency of the pitch-height stereotype, whereas variability in the latter stemmed first-and-foremost from individual differences in the generation of auditory images.

In sum, the current findings imply that the relative potency of exogenous and endogenous response priming can vary within an individual even when these two types of priming appear to be equipotent in group averages. Thus, the degree to which the perception of a stimulus primes movement in an individual is not necessarily predictive of how strongly the same movement would be primed if the person were to imagine this stimulus when it is absent. This seems to be somewhat inconsistent with the notion that perception and mental imagery play functionally equivalent roles in action planning and control.

It is a challenge for future research to account for the independent effects of exogenous and endogenous response priming observed in the current study. Perhaps the methods of neuroscience will allow this challenge to be met. Perception and mental imagery are clearly separable at some level of analysis in the brain. Research in neuropsychology 
has revealed dissociations between visual perception and imagery, where brain damage impairs one process while sparing the other (Bartolomeo, 2002). Furthermore, neuroimaging studies of healthy individuals have shown that the overlap in brain areas recruited during visual perception and imagery is "neither complete nor uniform" (Ganis, Thompson, \& Kosslyn, 2004, p. 226). This also applies in the case of perception and imagery in the auditory modality. Although secondary (associative) auditory cortical areas are activated during both perception and imagery, primary (sensory) auditory cortical areas are apparently not implicated in imagery (Zatorre, Halpern, Perry, Meyer, \& Evans, 1996). Furthermore, the supplementary motor area (SMA) is more strongly activated during auditory imagery than during auditory perception, which suggests that the generation of auditory images may involve motor processes such as subvocalization, e.g., inner singing of pitches (Brodsky, Henik, Rubinstein, \& Zorman, 2003; Halpern, Zatorre, Bouffard, \& Johnson, 2004).

Perhaps light can be shed on the relationship between exogenous and endogenous auditory priming by examining the correlation between SRC and REC effect magnitudes and activity in the primary auditory cortex and the SMA. The SMA is particularly intriguing in this regard, as it has been hypothesized to play a special role in the planning of 'voluntary' movements (i.e., those that are self-initiated rather than externally-triggered) (e.g., Cunnington, Windischberger, Deecke \& Moser, 2003; Deecke \& Lang, 1990; Tanji \& Mushiake, 1996). It is an open question whether SMA activity differentiates between endogenous and exogenous response priming.

\section{Acknowledgements}

We thank Yi Zhang for running the experiment. We also thank Dieter Nattkemper, Bruno Repp, and an anonymous reviewer for helpful comments on an earlier version of the manuscript. A preliminary version of the results was reported at the 45. Tagung experimentell arbeitender Psychologen, Kiel, Germany, March, 2003 (Keller \& Koch, 2003).

\section{References}

Adam, J. J., Boon, B., Paas, F. G. W., \& Umiltà, C. (1998). The up-right/down-left advantage for vertically oriented stimuli and horizontally oriented responses: A dual-strategy hypothesis. Journal of Experimental Psychology: Human Perception \& Performance, 24, 1025-1039.
Bartolomeo, P. (2002). The relationship between visual perception and visual mental imagery: A reappraisal of the neuropsychological evidence. Cortex, 38, 357-378. $\mid w w w$

Brodsky, W., Henik, A., Rubinstein, B., \& Zorman, M. (2003). Auditory imagery from musical notation in expert musicians. Perception \& Psychophysics, 65, 602-612. WWW

Cunnington, R., Windischberger, C., Deecke, L., \& Moser, E. (2003). The preparation and readiness for voluntary movement: a high-field event-related fMRI study of the Bereitschafts-BOLD response. NeuroImage, 20, 404-412. $\overline{\mathrm{WWw}}$

De Jong, R., Liang, C. C., \& Lauber, E. (1994). Conditional and unconditional automaticity: A dual-process model of effects of spatial stimulusresponse correspondence. Journal of Experimental Psychology: Human Perception \& Performance, 20, 731-750 www

Deecke, L., \& Lang, W. (1990). Movement-related potentials and complex actions: coordinating role of the supplementary motor area. In J. C. Eccles \& O. Creutzfeldt (Eds.), The principles of design and operation of the brain (pp. 303-336). Berlin: Springer.

Elsner, B., \& Hommel, B. (2001). Effect anticipation and action control. Journal of Experimental Psychology: Human Perception and Performance, 27, 229-240. WWw

Elsner, B., \& Hommel, B. (2004). Contiguity and contingency in the acquisition of action effects. Psychological Research, 68, 138-154. Www

Elsner, B., Hommel, B., Mentschel, C., Drzezga, A., Prinz, W., Conrad, B., \& Siebner, H. (2002). Linking actions and their perceivable consequences in the human brain. Neuroimage, 17, 364-372. Www

Finke, R. A. (1980). Levels of equivalence in imagery and perception. Psychological Review, 87, 113-132.

Finke, R. A. (1985). Theories relating imagery to perception. Psychological Bulletin, 98, 236-259. |www

Fitts, P. M., \& Deininger, R. L. (1954). S-R compatibility: Correspondence among paired elements within stimulus and response codes. Journal of Experimental Psychology, 48, 483-492. WwW

Ganis, G., Thompson, W. L., \& Kosslyn, S. M. (2004). Brain areas underlying visual imagery and visual perception: an fMRI study. Cognitive Brain Research, 20, 226-241. WWW

Greenwald, A. G. (1970). Sensory feedback mechanisms in performance : With special reference to the ideo-motor mechanism. Psychological Review, 77, 73-99. Www 
Halpern, A. R., Zatorre, R. J., Bouffard, M., \& Johnson, J. A. (2004). Behavioral and neural correlates of perceived and imagined timbre. Neuropsychologia, 42, 1281-1292. $\underline{W w \mid}$

Harleß, E. (1861). Der Apparat des Willens [The mechanism of will]. Zeitschrift für Philosophie und philosophische Kritik, 38, 50-73.

Hoffmann, J., Stoecker, C., \& Kunde, W. (2004). Anticipatory control of actions. International Journal of Sport and Exercise Psychology, 2, 346-361.

Hommel, B., Müsseler, J., Aschersleben, G., \& Prinz, W. (2001). The theory of event coding (TEC): A framework for perception and action. Behavioral \& Brain Sciences, 24, 849-937. |www

Hommel, B., \& Prinz, W. (1997). (Eds.). Theoretical issues in stimulus-response compatibility. Amsterdam: North-Holland.

Hubbard, T. L., \& Stoeckig, K. (1992). The representation of pitch in musical images. In D. Reisberg (Ed.), Auditory imagery (pp. 199-236). Hillsdale, NJ: Lawrence Erlbaum.

Intons-Peterson, M. J. (1992). Components of auditory imagery. In D. Reisberg (Ed.), Auditory imagery (pp. 45-72). Hillsdale, NJ: Lawrence Erlbaum.

James, W. (1890). Principles of psychology. New York: Holt.

Keller, P., \& Koch, I. (2003). Stimulus-Reaktions und Reaktions-Effekt Kompatibilität zwischen Tonhöhe und räumlicher Höhe [Stimulus-response and response-effect compatibility between auditory pitch and spatial height]. Poster presented at 45. Tagung experimentell arbeitender Psychologen. Kiel, Germany, 24-26 March, 2003.

Koch, I., Keller, P. E., \& Prinz, W. (2004). The ideomotor approach to action control: Implications for skilled performance. International Journal of Sport and Exercise Psychology, 2, 362-375.

Koch, I., \& Kunde, W. (2002). Verbal response-effect compatibility. Memory \& Cognition, 30, 1297-1303. WWW

Kornblum, S., Hasbroucq, T., \& Osman, A. (1990). Dimensional overlap: Cognitive basis for stimulusresponse compatibility: A model and taxonomy. Psychological Review, 97, 253-270. |www|

Kosslyn, S. M., Brunn, J. L., Cave, K. R., \& Wallach, R. W. (1984). Individual differences in visual imagery: a computational analysis. Cognition, 18, 195-243.

Kosslyn, S. M., Ganis, G., \& Thompson, W. L., (2001). Neural foundations of imagery. Nature Reviews: Neuroscience, 2, 635-642. WwW

Kunde, W. (2001). Response-effect compatibility in manual choice reaction tasks. Journal of
Experimental Psychology: Human Perception \& Performance, 27, 387-394. [www]

Kunde, W. (2003). Temporal response-effect compatibility. Psychological Research, 67, 153-159.

Kunde, W. (2004). Response priming by supraliminal and subliminal action effects. Psychological Research, 68, 91-96. Www

Kunde, W., Koch, I., \& Hoffmann, J. (2004). Anticipated action effects affect the selection, initiation, and execution of actions. Quarterly Journal of Experimental Psychology, 57A, 87-106. WwW

Lidji, P., Kolinsky, R., Lochy, A., Karnas, D., \& Morais, J. (2005). Spatial response codes for musical stimuli: A piano in the head? Manuscript submitted for publication.

Lotze, R. H. (1852). Medicinische Psychologie oder die Physiologie der Seele [Medical psychology or the physiology of the soul]. Leipzig: Weidmann'sche Buchhandlung.

Masson, M. E. J., \& Loftus, G. R. (2003). Using confidence intervals for graphically based data interpretation. Canadian Journal of Experimental Psychology, 57, 203-220. www

Melara, R. D., \& Marks, L. E. (1990). Processes underlying dimensional interactions: Correspondences between linguistic and nonlinguistic dimensions. Memory \& Cognition, 18, 477-495. WwW

Neuhoff, J. G., Knight, R., \& Wayand, J. (2002). Pitch change, sonification, and musical expertise: Which way is up? Proceedings of the 2002 International Conference on Auditory Display, Kyoto, Japan, 2-5 June, 2002.

Prinz, W. (1987). Ideo-motor action. In H. Heuer \& A. F. Sanders (Eds.), Perspectives on perception and action (pp. 47-76). Hillsdale, NJ: Lawrence Erlbaum.

Proctor, R. W., \& Reeve, T. G. (Eds.) (1990). Stimulusresponse compatibility: An integrated perspective. Amsterdam: North-Holland.

Ratcliff, R. (1979). Group reaction time distributions and an analysis of distribution statistics. Psychological Bulletin, 86, 446-461. WWW

Richardson, J. T. E. (1999). Mental imagery. Psychology Press: Hove, U.K.

Rusconi, E., Kwan, B., Giordano, B. L., Umiltá, C., \& Butterworth, B. (2006). Spatial representation of pitch height: the SMARC effect. Cognition, 99, 113-129. |WwW|

Simon, J. R. (1969). Reaction toward the source of stimulus. Journal of Experimental Psychology, 81, 174-176.

Simon, J. R., Mewaldt, S. P., Acosta, E., \& Hu., J.-M. (1976). Processing auditory information: Interaction 
of two population stereotypes. Journal of Applied Psychology, 61, 354-358. WwW

Tanji, J., \& Mushiake, H. (1996). Comparison of neuronal activity in the supplementary motor area and primary motor cortex. Cognitive Brain Research, 3, 143-150.

Tlauka, M., \& McKenna, F. P. (1998). Mental imagery yields stimulus-response compatibility. Acta Psychologica, 98, 67-79. |WWw

Walker, R. (1987). The effects of culture, environment, age, and musical training on choices of visual metaphors for sound. Perception \& Psychophysics,

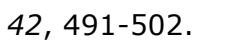

Wiegand, K., \& Wascher, E. (2005). Dynamic aspects of stimulus-response correspondence: Evidence for two mechanisms involved in the Simon effect. Journal of Experimental Psychology: Human Perception \& Performance, 31, 453-464. WwW

Zatorre, R. J., Halpern, A. R., Perry, D. W., Meyer, E., \& Evans, A. C. (1996) Hearing in the mind's ear: A PET investigation of musical imagery and perception. Journal of Cognitive Neuroscience, 8, 29-46.

Ziessler, M., Nattkemper, D., \& Frensch, P. (2004). The role of anticipation and intention in the learning of effects of self-performed actions. Psychological Research, 68, 163-175. WWW 